\title{
Ion beam induced nanosized Ag metal clusters in glass
}

\author{
H.-E. Mahnke ${ }^{\text {a,* }}$, B. Schattat ${ }^{\text {a }}$, P. Schubert-Bischoff ${ }^{a}$, N. Novakovic ${ }^{\text {a,b }}$ \\ ${ }^{a}$ Hahn-Meitner-Institut Berlin GmbH, Ionenstrahllabor ISL, Bereich Strukturforschung, Glienicker Strasse 100, D-14109 Berlin, Germany \\ ${ }^{\mathrm{b}}$ VINČA, POB 522, 11001 Belgrade, Serbia and Montenegro
}

Available online 27 December 2005

\begin{abstract}
Silver metal clusters have been formed in soda lime glass by high-energy heavy-ion irradiation at ISL. The metal cluster formation was detected with X-ray absorption spectroscopy (EXAFS) in fluorescence mode, and the shape of the clusters was imaged with transmission electron microscopy. While annealing in reducing atmosphere alone, leads to the formation of metal clusters in Ag-containing glasses, where the Ag was introduced by ion-exchange, such clusters are not very uniform in size and are randomly distributed over the Ag-containing glass volume. Irradiation with $600-\mathrm{MeV}$ Au ions followed by annealing, however, results in clusters more uniform in size and arranged in chains parallel to the direction of the ion beam.
\end{abstract}

(c) 2005 Elsevier B.V. All rights reserved.

PACS: $61.10 . \mathrm{Ht} ; 61.80 . \mathrm{Jh} ; 68.37 . \mathrm{Lp} ;$ 78.55.Qr

Keywords: XAFS; TEM; Local structure; Nanoclusters; Ion beam modification; Ion tracks

\section{Introduction}

Glasses containing metal clusters have attracted quite some attention both in cluster research and in possible applications of such clusters for magnetic or optoelectronic purposes. So nanometer-sized clusters of noble metals in glasses exhibit strong absorption of visible light which, in addition, may be highly polarization dependant depending on size and shape with special alignment of the clusters $[1,2]$. Various preparation methods are pursued to obtain control of the mechanisms to form such clusters. A promising approach is the irradiation of glasses containing the wanted metal as a metal oxide with heavy-ion beams at $\mathrm{MeV}$ energies. This procedure has been investigated in the case of soda lime glass with $\mathrm{Cu}$ clusters from $\mathrm{Cu}_{2} \mathrm{O}$ in the original mixture of the oxides [3] and with $\mathrm{Ag}$ clusters from the incorporation by ion-exchange $[4,5]$. In both cases, metal clusters were already produced by other means

\footnotetext{
* Corresponding author. Tel.: +49 308062 2715; fax: +49 3080622293. E-mail address: mahnke@hmi.de (H.-E. Mahnke).
}

of treatment, such as heat treatment in reducing atmosphere and/or laser irradiation. By using ion beams, it could be shown that the special energy deposition due to the electronic stopping of heavy ions may be advantageous to the cluster size distribution (see the detailed discussion in [3]). Furthermore, irradiation with ion beams with considerable electronic stopping power results in alignment effects of the clusters along the ion beam direction. This was demonstrated by $30-\mathrm{MeV} \mathrm{Si}$ ion irradiation of $\mathrm{Ag}$ clusters, which were nucleated with $1-\mathrm{MeV}$ Xe irradiation at high fluences [5]. Similarly, modification of size and shape (elongation) have been observed for Co nanoparticles in $\mathrm{SiO}_{2}$ by $200-\mathrm{MeV}^{127}$ I ion irradiation [6]. These studies prompted us to investigate metal cluster formation in glasses with ions of very high electronic stopping power with the possibility of forming metal wires along the ion tracks, hundreds of nanometer, up to micrometers long, with diameters of the typical size of an ion track of some nanometer.

Access to the understanding of the cluster formation can be obtained by methods sensitive to the local structure which is not obscured when only nanometer-sized objects are investigated. Such a technique is provided by the 